\title{
Numerical and Experimental Modeling of a T-joint Fillet Welding Process
}

\author{
by Z. Tonković*, M. Perić**, M. Surjak*, I. Garašić*, I. Boras*, A. Rodić*, S. Švaić* \\ ^Faculty of Mechanical Engineering and Naval Architecture, University of Zagreb, I. Lučića 5, 10000 Zagreb, \\ Croatia, \{zdenko.tonkovic, martin.surjak, ivica.garasic, ivanka.boras, alan.rodic, srecko.svaic\}@fsb.hr \\ **Bureau of Energetics and Mechanical Engineering Ltd., Petrovaradinska 7, 10000 Zagreb, Croatia, \\ mato.peric@fsb.hr
}

\begin{abstract}
This paper presents a numerical and experimental study of residual stresses and distortions induced by the Tjoint welding of two plates. Within the framework of numerical investigations a thermo-mechanical finite element analysis is performed by using a shell/three-dimensional modeling technique to improve both the computational efficiency and the accuracy. The influence of the choice of the local 3D model size on the temperature distribution, residual stresses and displacements is investigated. In order to validate numerical model, a series of experiments using fully automated welding process are conducted. Thermographic camera and optical measurement system is used to measure temperature and displacement distributions.
\end{abstract}

\section{Introduction}

It is well known that during the welding process, due to localized heating and subsequent rapid cooling, residual stresses appear around welding zones and cause post-weld deformations of the structure. Numerical simulations have become an important tool to predict these phenomena, but the structures that can be simulated and the time period over which they can be studied will always be limited by computer power.

In principle, a finite element (FE) simulation of the welding process consists of two main parts: thermal analysis and mechanical stress analysis. In thermal analysis, the temperature field is determined as a function of time for each integration point. This temperature time-history is used as an input into the thermal stress analysis. Herein, the thermal solution can be sequentially or fully coupled with the mechanical solution of the structure. Because the rate of heat generation due to mechanical dissipation energy can be neglected in the heat transfer analysis, a sequentially coupled thermal-stress analysis is commonly applied for the simulation of a welding process in which a thermal analysis is followed by a stress analysis [1-4]. As presented in the literature, the use of three-dimensional (3D) models is required for accurate prediction of post-weld deformation and residual stresses distribution. Such a complex model can be computationally expensive or even oversized for available resources [5].

To reduce the simulation time and simultaneously maintain the accuracy, this study approached towards numerical simulation of welding process by using a sequential method combining 3D solid and shell elements. All computations have been performed within the FE software Abaqus/Standard [6]. Three-dimensional continuum elements are used close to the weld where stress gradients are high; while outside the welding zone, the structure is discretized with shell elements to reduce the overall model size. Thus, this shell/3D modeling technique combines the accuracy of the full three-dimensional solution with the computational efficiency of a shell finite element model. For verification of the shell-to-solid coupling model, example of a T-joint fillet welding is chosen. The welding experiments and thermographic imaging are performed and the test results are compared to those obtained from finite element analyses.

\section{Experimental investigations}

\subsection{Welding conditions}

The geometry of two plates welded into a T-shape including the relevant dimensions, is presented in Fig. $1 \mathrm{a}$. The plates are made of nonalloyed structural steel (DIN St.52-3) and welded by fillet welds with $7 \mathrm{~mm}$ leg. Joint preparation is suited for double sided fillet weld with partial penetration with no gap between horizontal and vertical plate. Positioning angle between horizontal and vertical plate is $90^{\circ}$. The MAG (135) process is used. Welding of fillet welds is performed by 6 axis robot supplied with digital inverter power source in order to ensure the process stability. Welding parameters chosen for this analysis were as follows: wire diameter $1.2 \mathrm{~mm}$, shielding gas composition $82 \% \mathrm{Ar} / 18 \% \mathrm{CO}_{2}$, welding current $I=270 \mathrm{~A}$, arc voltage $U=29 \mathrm{~V}$ and welding speed $v=400 \mathrm{~mm} / \mathrm{min}$. Welding sequence and direction are presented in Figure 1. After welding of the first fillet weld the welding jig is rotated for $180^{\circ}$ and prepared for the second pass. The time period between the end of the first pass and the beginning of second pass was 214 seconds. For the first and second pass welding parameters remained same without any corrections. Material transfer during MAG welding is supported by pulse welding current through which short circuiting and spattering were avoided. 


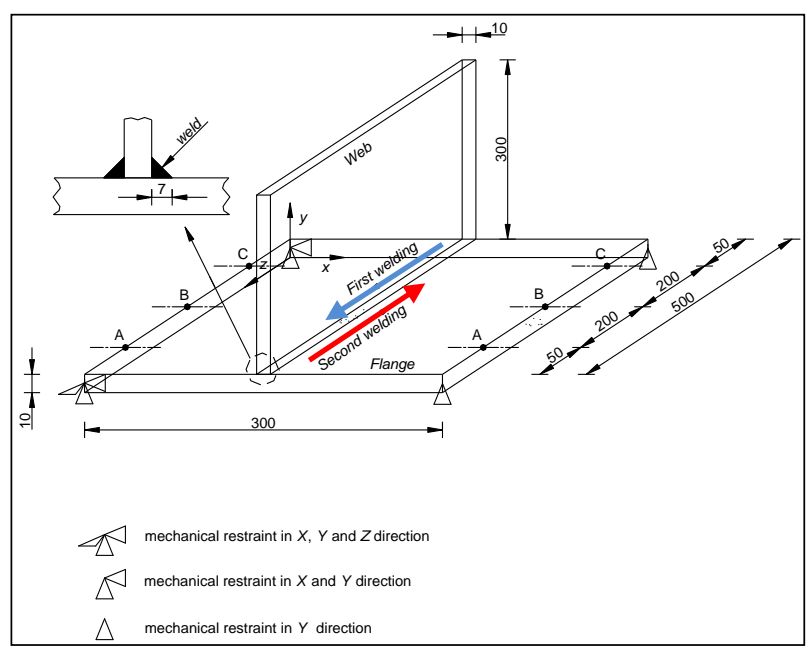

a)

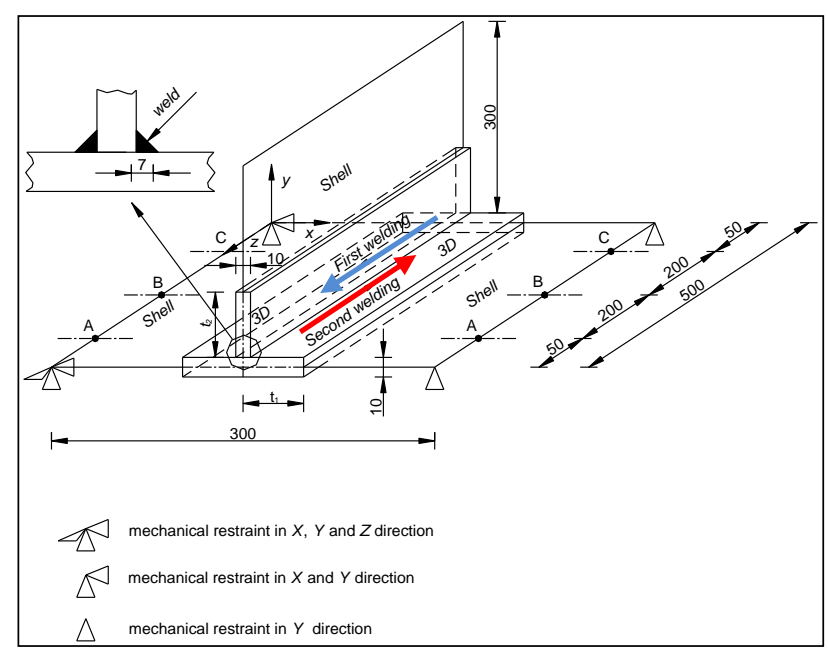

b)

Fig. 1. Geometry of the T- joint: a) the full $3 D$ model, b) the shell/3D model

\subsection{Thermal measurements}

Surface temperature fields of the two plates were captured during T-joint welding using IR camera. Analysis of the obtained thermograms allowed for the evaluation of the numerical model as well as for determination of time reference enabling the comparison of the data logged from different instruments.

Furthermore, in order to evaluate obtained thermograms, temperature measurements are simultaneously conducted by thermocouples (Type $\mathrm{K}$ ) which were $6 \mathrm{~mm}$ stepped in the material, at the bottom side of horizontal plate. The location of thermocouples is shown in Figure 2.

The recording process is conducted with pauses involved between three stages of the experiment consisting of the two passes of the electrode and cooling stage. The time increment for recording of thermograms is differently adopted for the first two stages ( 2 seconds) and the last - cooling stage of the experiment.

Particular analysis of thermograms is performed for specific time instants (Fig. 3.) The results obtained from IR imaging are compared with those from the numerical simulations for the selected profile (profile-1) which is presented in Fig. 2. and Fig 3.

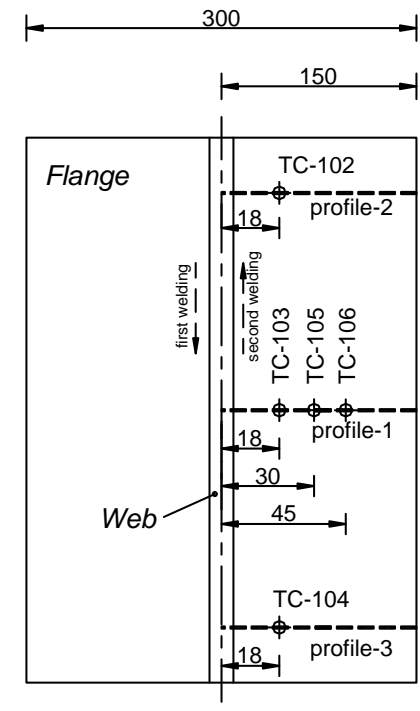

$\phi$ Thermocouple

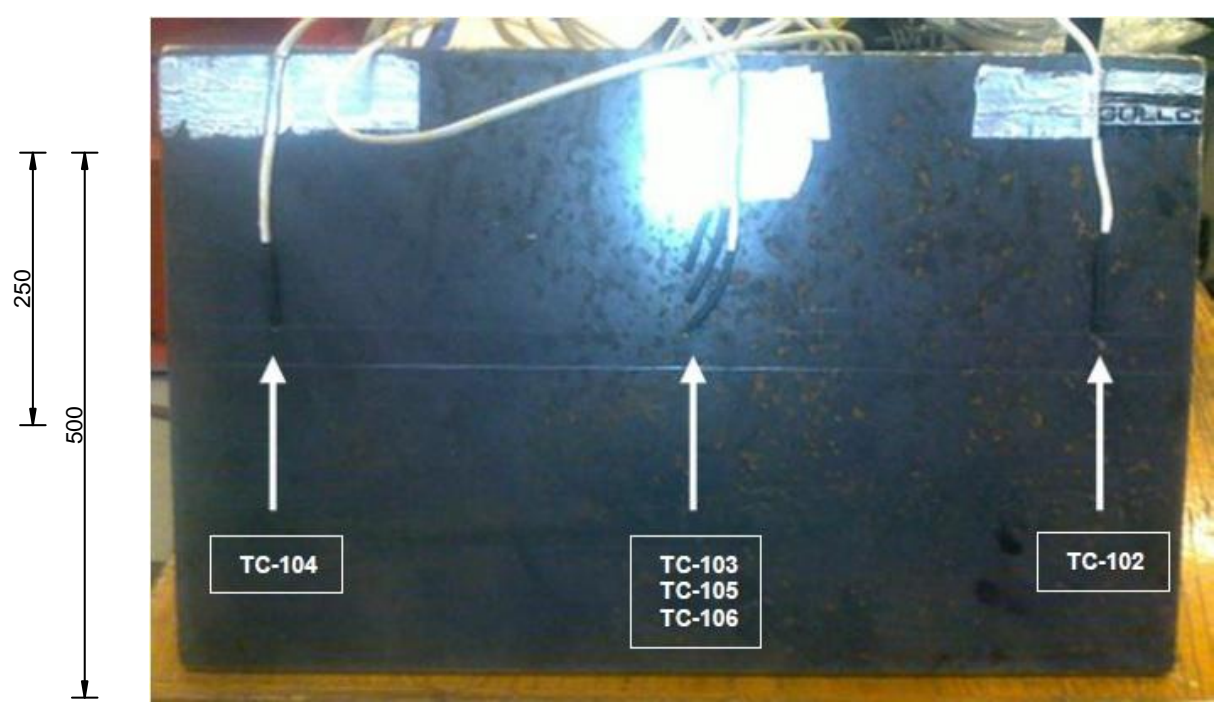

Fig. 2. Locations of the thermocouples 

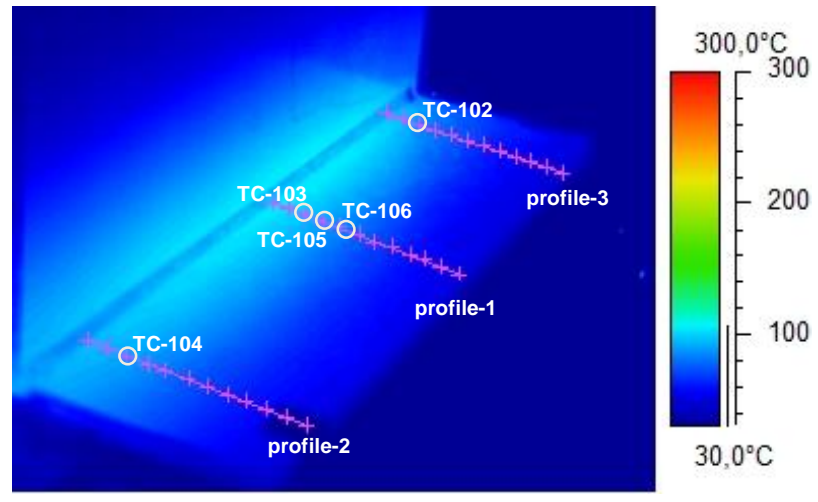

a)

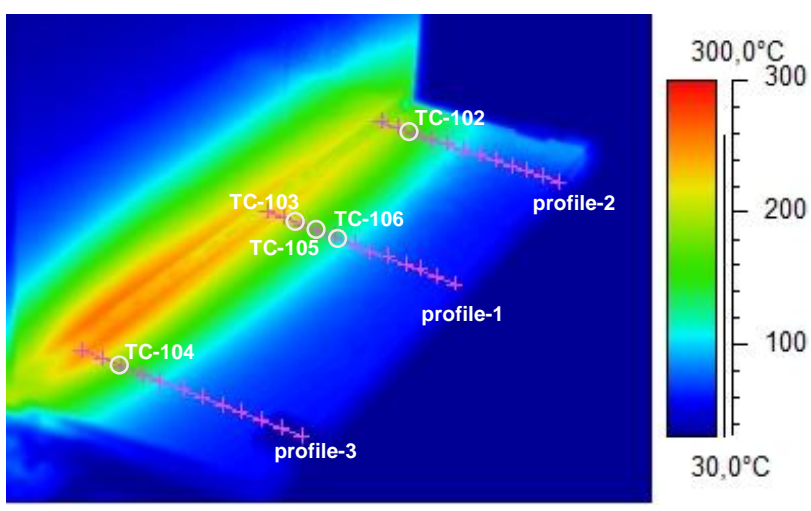

b)

Fig. 3. Thermograms; a) $290 \mathrm{~s}$ after the beginning of the welding process and b) $403 \mathrm{~s}$ after the beginning of the welding process

\subsection{Displacement measurements}

The displacements of the plates during welding are measured by using non-contact 3D image correlation systems ARAMIS 4M for deformation analysis and synchronized with the thermographic measuring to find correlation between displacement of certain points on the plate and temperature. In this case two cameras are used that are calibrated prior to measuring. Before the start of the experiment the specimen is prepared by applying a random spray pattern over the mat surface in order to clearly allocate the pixels in the camera images. To get the good raster in this experiment the mixture of titanium oxide and alcohol compressed to 1 bar is applied on the plate surface with powder spray. After recording the series of images the optical system Aramis detects the deformation of the specimen trough the images by means of various square or rectangular facets. From each valid facet, a measuring point results after computation. In this measurement squared $15 \times 15$ pixel facets are used. The welds on both sides of the plates are done in sequence, one after the other and the optical system Aramis is always measuring from the opposite side of the welding arc so the plates needed to be rotated for $180^{\circ}$ between each welding pass. According to that white markers are glued near the specimen that are later used as a reference points for data transformation. The default coordinate system is usually fixed with the Aramis. In this case the new coordinate system is defined by reference points. The strain and displacement data of a measuring project are transformed into a new coordinate system in order to be interpreted correctly. The full field displacement distribution is shown in Fig. 4.

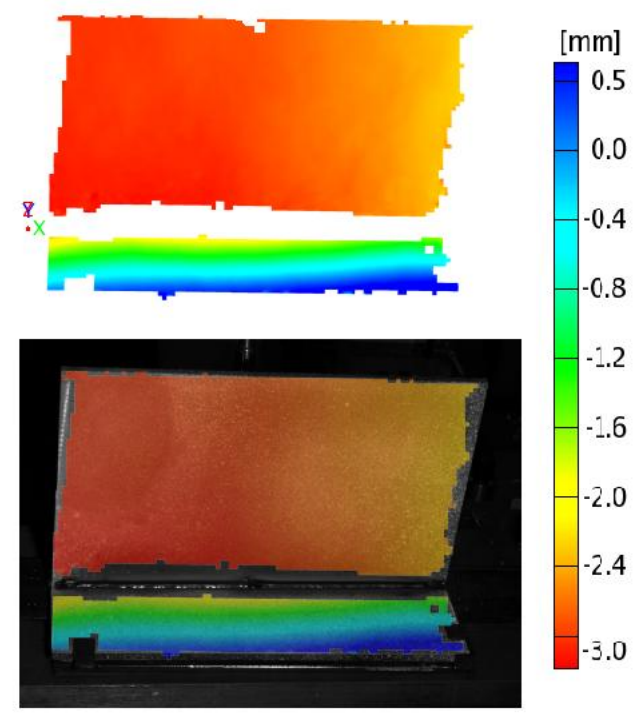

Fig. 4. Measured displacement distribution 


\section{Numerical modeling}

Within the framework of numerical investigations two models are constructed: a full 3D model (Fig. 1a) and a combined shell/3D finite elements model (Fig. 1b). For the numerical simulation of the welding process using the combination of the $3 \mathrm{D}$ and the shell elements several different models are analyzed. They differed in the size of the structure discretized with 3D continuum elements. More details concerning the applied shell/3D modeling technique can be found in authors' paper [5]. Due to conciseness of this paper, the numerical results are presented only for the T30 $\times 33.7$ shell/3D model $\left(t_{1}=30 \mathrm{~mm}, t_{2}=33.7 \mathrm{~mm}\right)$ which demonstrates a good match for deflections and stresses in comparison with the full 3D model. To discretize the full 3D geometry, DC3D8 elements are applied for the thermal model, and C3D8 elements for the mechanical model. Herein, the size of elements becomes larger as the distance from the root of the weld increases as shown in Fig. $7 \mathrm{a}$ presenting the finite element mesh. The 3D mesh consists of 22176 elements. To discretize the shell/3D geometry DC3D8 solid elements and DS4 shell elements are used during the thermal analysis, while for the mechanical analysis, C3D8 solid elements and S4 shell elements are used. The part of the plate closest to the weld is discretized with 3D continuum elements using the same elements as in the full 3D model (Fig. 5b). Presented global shell model contains 2652 S4 elements, while the submodel is discretizated with 9240 C3D8 elements.

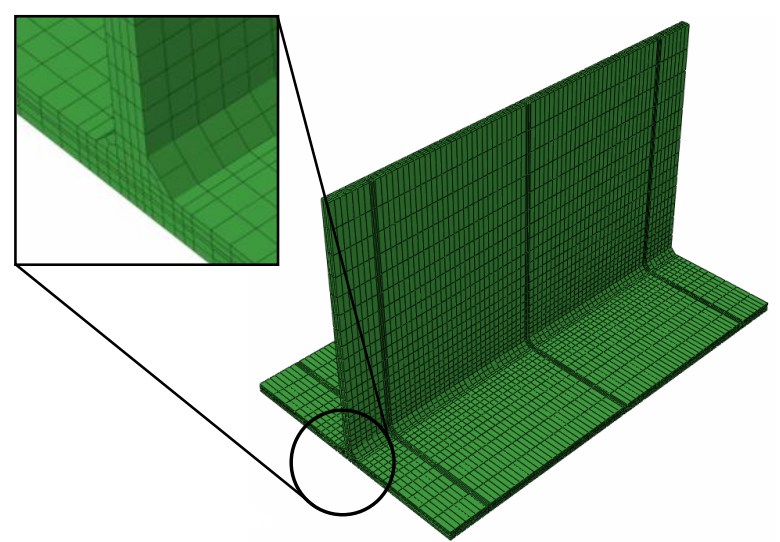

a)

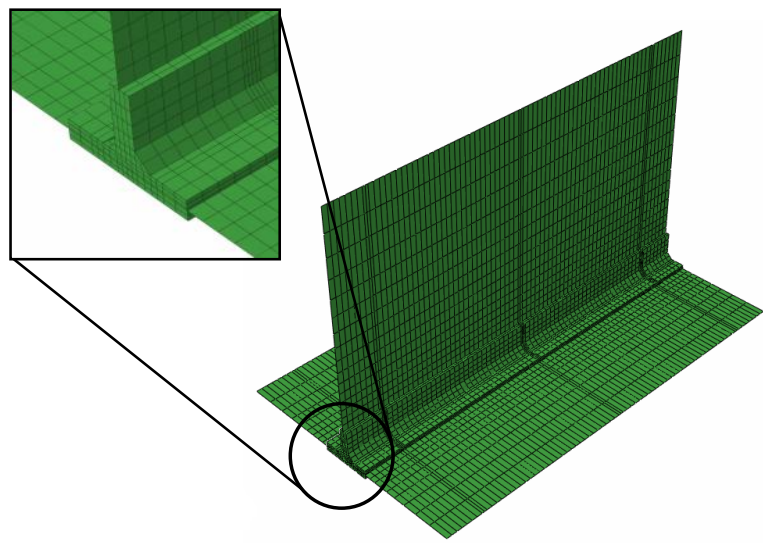

b)

Fig. 5. Typical FE meshes: a) full 3D, b) combined shell/3D

Boundary conditions which are applied are schematically shown in Figure 1. Mechanical and thermal properties of St.52-3 steel depending on temperature are shown in Figures 6 and 7. The analysis is made with the assumption that the base metal and weld metal have the same mechanical properties. Non-linear material behavior is modeled using incremental plasticity with the von Mises yield criterion, together with the associated flow rule and assuming large deformations. The material is modeled as an elastic - plastic solid with isotropic and kinematic hardening. The governing equation for transient non-linear heat transfer analysis is:

$$
\frac{\partial}{\partial x}\left(k_{x} \frac{\partial T}{\partial x}\right)+\frac{\partial}{\partial y}\left(k_{y} \frac{\partial T}{\partial y}\right)+\frac{\partial}{\partial z}\left(k_{z} \frac{\partial T}{\partial z}\right)+Q=\rho C \frac{\partial T}{\partial t}
$$

where, $k_{x}, k_{y}, k_{z}$ are the thermal conductivities in the $x, y$ and $z$ directions respectively, $T$ is the current temperature, $Q$ is the heat generation, $\rho$ is the density, $C$ is the specific heat capacity and $t$ is the time, respectively. General solution of equation (1) is obtained introducing the initial and boundary conditions, as follows:

Initial condition:

$$
T(x, y, z, 0)=T_{0}(x, y, z)
$$

Boundary conditions:

$$
\left(k_{\mathrm{x}} \frac{\partial T}{\partial x} N_{x}+k_{y} \frac{\partial T}{\partial y} N_{y}+k_{z} \frac{\partial T}{\partial z} N_{z}\right)+q_{s}+h_{c}\left(T-T_{\infty}\right)+h_{\mathrm{r}}\left(T-T_{\mathrm{r}}\right)=0
$$

where, $N_{x}, N_{y}, N_{z}$ are the direction cosine normal to the boundary, $h_{c}$ and $h_{r}$ are the convection and radiation heat transfer coefficients respectively, $q_{s}$ is the boundary heat flux and $T_{r}$ is the temperature of radiation heat source and $T_{\infty}$ is the surrounding temperature. Radiation heat losses are dominant near the weld and can be expressed by equation:

$$
h_{\mathrm{r}}=\sigma \varepsilon F\left(T^{2}+T_{\mathrm{r}}^{2}\right)\left(T+T_{\mathrm{r}}\right)
$$


where, $\sigma=5.67 \times 10^{-8} \mathrm{~J} /\left(\mathrm{m}^{2} \mathrm{~K}^{4}\right)$ is the Steffan-Boltzman constant, $\varepsilon$ is the effective emissivity and $F$ is the configuration factor. The total heat input is given by:

$$
q=\frac{\eta U I}{V_{H}}
$$

where $\eta$ is the arc efficiency, $I$ is current, $U$ is the arc voltage and $V_{H}$ is volume of the activated weld bead element, respectively.

The following data is assumed: the convection heat transfer coefficient $h_{c}=10 \mathrm{~W} / \mathrm{m}^{2} \mathrm{~K}$, the efficiency of the heat input $E_{\mathrm{ff}}=85 \%$ and the emissivity $\varepsilon=0.9$. Heat flux applied to the weld was uniform, $q=3.994 \times 10^{10} \mathrm{~J} / \mathrm{m}^{3} \mathrm{~s}$. All computations have been performed within the FE software Abaqus/Standard [6].

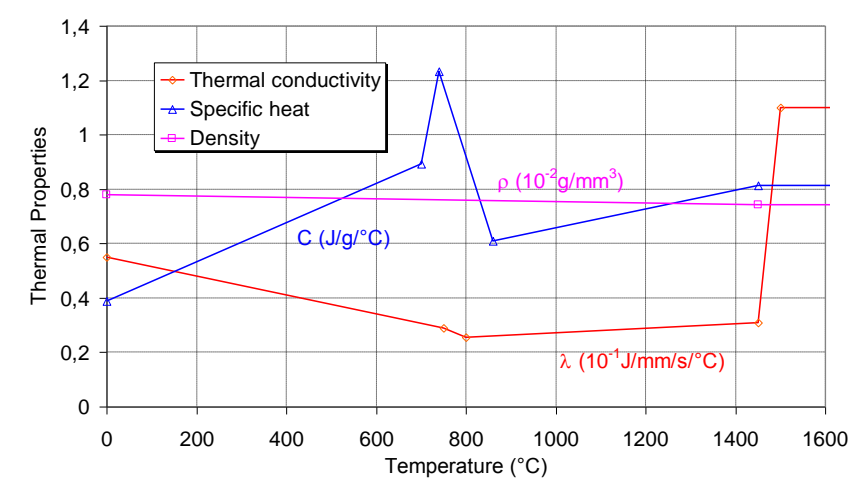

Fig. 6. Thermal properties of St.52-3 steel [8]

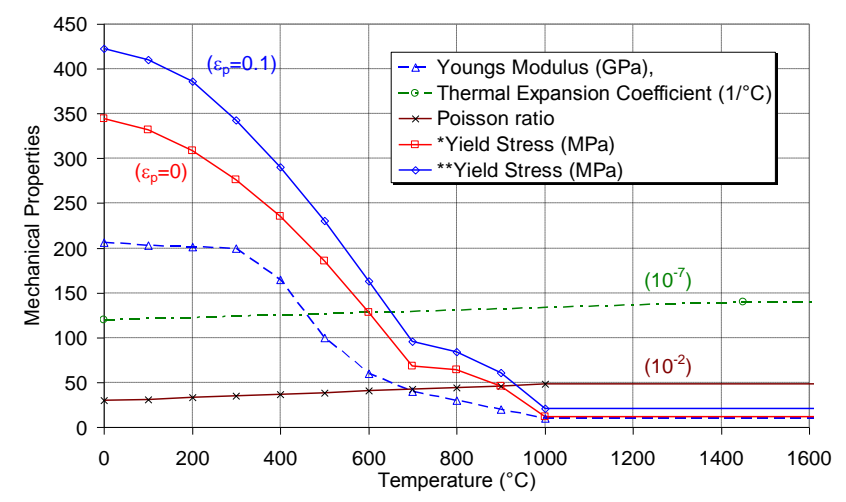

Fig. 7. Mechanical properties of St.52-3 steel [8]

\section{Comparison of experimental and numerical results}

\subsection{Thermal analysis}

Fig. 8 shows the comparison between temperature profiles obtained using the thermocouples and IR thermographic camera $290 \mathrm{~s}$ i $403 \mathrm{~s}$ after the beginning of the welding process at the locations of installed thermocouples. The associated numerical results are presented in Fig. 9. Comparison between temperature profiles obtained using the thermocouples, IR thermographic camera and finite element method $290 \mathrm{~s}$ and $403 \mathrm{~s}$ after the beginning of the welding process are shown in Fig. 10. As may be seen from figures, the results obtained by the IR thermographic camera are in good agreement to results observed experimentally. By comparing the results presented in Figs. 8 to 10 it is evident that the temperatures obtained by numerical simulation are somewhat lower than those obtained by experimental measurements. Besides, from the finite element results obtained by the shell/3D modeling technique and full 3D model, it is concluded that the size of the 3D submodel does not influence the temperature field distribution; therefore, the temperature field for all shell/3D models analyzed predicts the temperature field as well as the 3D model [5]. 


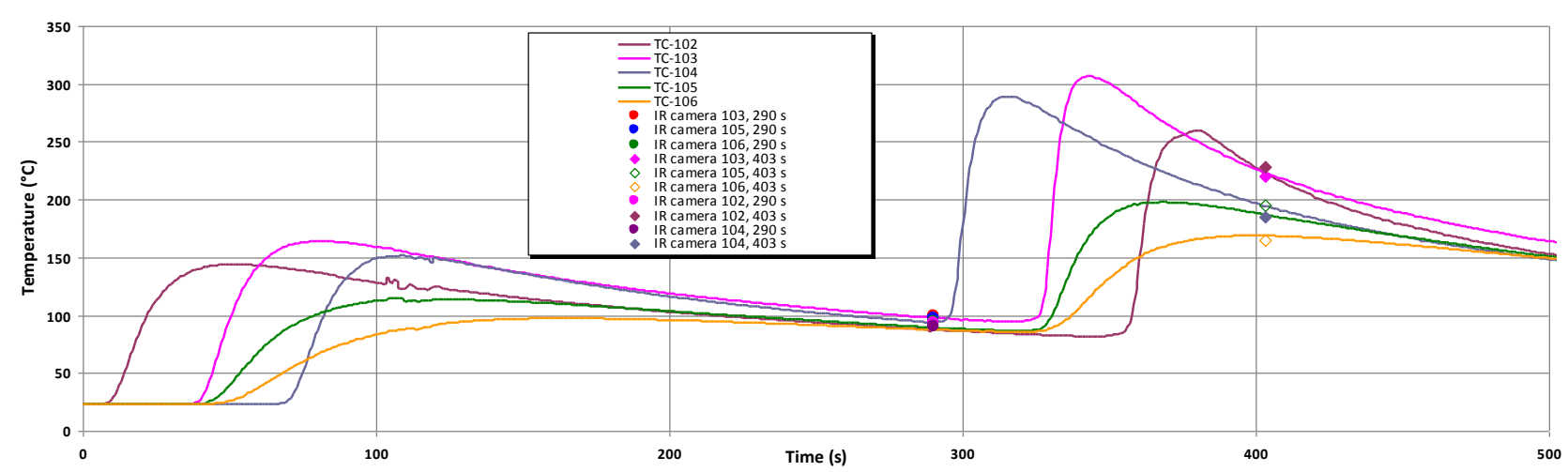

Fig. 8. Comparison between temperature profiles obtained using the thermocouples and IR thermographic camera $290 \mathrm{~s}$ and $403 \mathrm{~s}$ after the beginning of the welding process

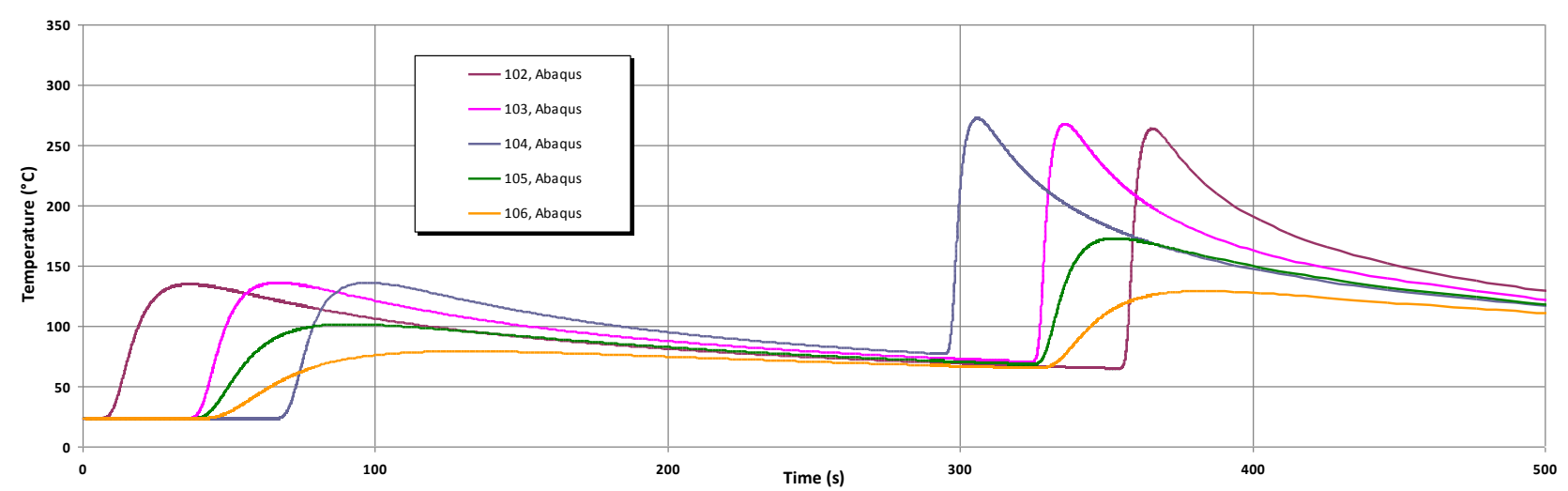

Fig. 9. Temperature profiles obtained using the finite element method $290 \mathrm{~s}$ and $403 \mathrm{~s}$ after the beginning of the welding process

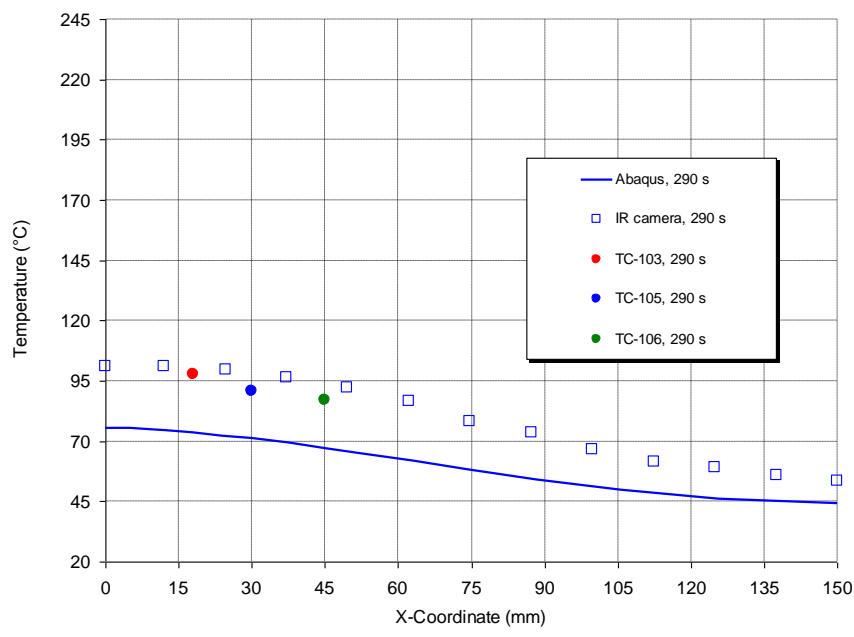

a)

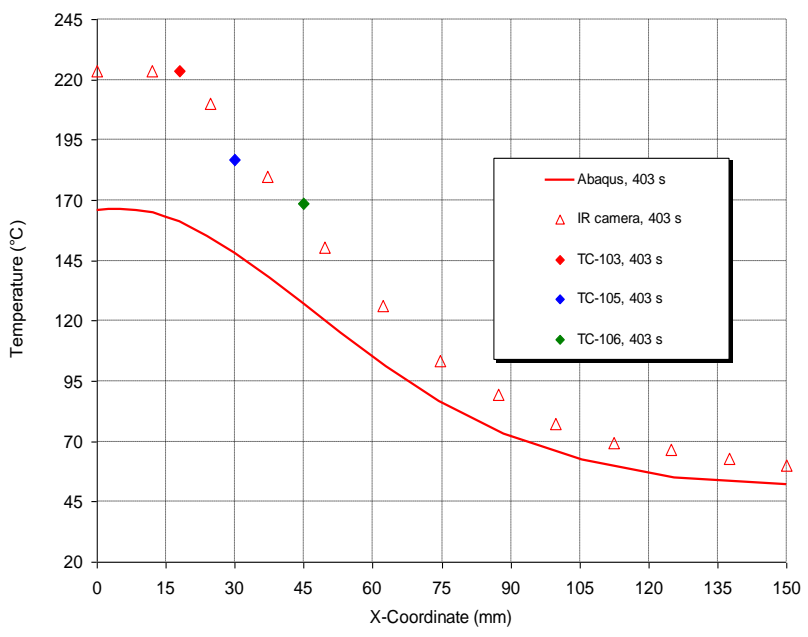

b)

Fig. 10. Comparison between temperature profiles obtained using the thermocouples, IR thermographic camera and finite element method; a) $290 \mathrm{~s}$ after the beginning of the welding process, b) $403 \mathrm{~s}$ after the beginning of the welding process 


\subsection{Mechanical analysis}

The comparison of the numerical and experimental results obtained by the optical system Aramis $4 \mathrm{M}$ for the plate deflections in the $y$ direction (along B-B line seen in Fig. 1) after welding and cooling process completion is given in Fig. 11a. Herein, the full 3D numerical results are compared with the results obtained by the shell/3D modeling technique. Good agreement with the numerical results as well as relatively little deviation from the experimental solutions is obtained. The residual stresses in the weld direction (along the line B-B seen in Fig. 1) on the upper surface of the flange are presented in Fig. 11b. Here should be mentioned that the same results are obtained using the full 3D finite element model or the shell/3D model.

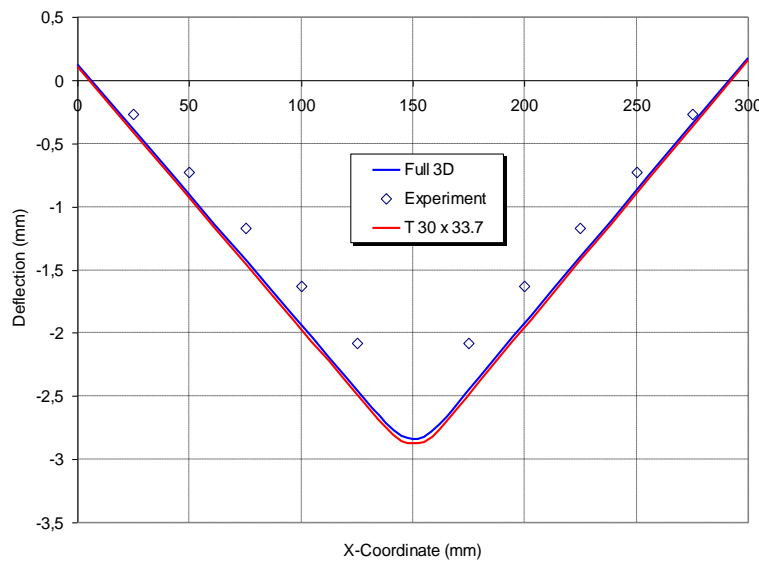

a)

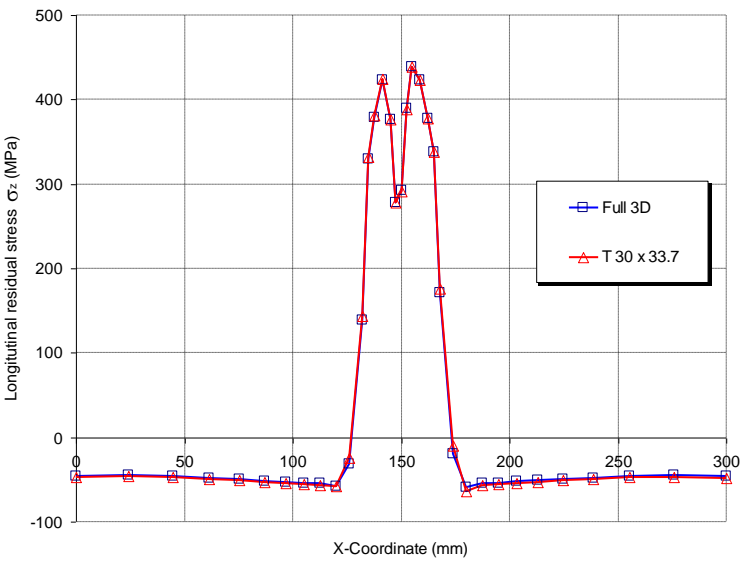

b)

Fig. 11. a) Deflections on the middle surface of the flange along the line $B-B$ seen in Fig. 1, b) Residual stresses in the weld direction (along the line B-B seen in Fig. 1, on the upper surface of the flange

\section{Conclusion}

Efficiency and accuracy of shell/3D technique for welding simulation is investigated. The influence of the 3D zone size on the convergence of the results is defined. The minimal 3D submodel size is selected on the basis of the error analysis. The size of the computational model is greatly reduced by adopting shell/3D analysis. The welding experiments are conducted and the measured temperatures and displacements are compared with results obtained by numerical analysis. It is shown that the numerical results agree well with the experimental results.

\section{REFERENCES}

[1] Deng D., Liang W., Murakawa H., "Determination of welding deformation in fillet-welded joint by means of numerical simulation and comparison with experimental measurements". Journal of Materials Processing Technology, vol. 183, pp. 219-225, 2007.

[2] Lee, C. H., Chang, K. H., "Three-dimensional finite element simulation of residual stresses in circumferential welds of steel pipe diameter effects". Material Science and Engineering, vol. 487, pp. 210-218, 2008.

[3] Deng D., "FEM prediction of welding residual stress and distortion in carbon steel considering phase transformation effects". Materials and Design, vol. 30, pp. 359-366, 2009.

[4] Gannon L., Liu Y., Pegg N., Smith M., "Effect of welding sequence on residual stress and distortion in flat-bar stiffened plates". Marine Structures, doi:10.1016/j.marstruc.2010.05.002, 2010.

[5] Perić, M., Tonković, Z., Karšaj, I. Numerical analysis of Residual Stresses in Welding Process Using Shell to Solid Coupling Technique, International Conference on Advances in Welding Science and Technology for Construction, Energy and Transportation Systems (AWST-2010), Istanbul, Turkey, 2010.

[6] Abaqus/Standard 6.10.1, Dassault Systemes, Simulia.

[7] Švaić, S., Boras, I., Application of control volume numerical method in thermographic analysis of relative material loss, QIRT'06, International Conference Quantitative InfraRed Thermography, Padova, Italy, CD Proceeding, 2006.

[8] Pilipenko A. Computer simulation of residual stress and distortion of thick plates in multi-elektrode submerged arc welding. Their mitigation techniques. Doctoral thesis, Trondheim. July 2001. 